\title{
Facilitation of seeing by hearing: Binaural summation*
}

\author{
GWENETH T. CAMPBELL and RICHARD L. TAYLOR \\ Memorial University, St. John's, Nfld., Canada
}

Ss made same/different RT judgments of digits presented successively on a CRT screen. A computer presented the test digit either to the left or right of fixation, and turned on a 90-dB SPL noise either monaurally left or right, or binaurally in S's earphones shortly after digit onset. Compared to quiet trials, RTs were fastest in the binaural condition, although all types of noisy trials were faster. Test digits shown to the right of fixation produced faster mean RTs overall, but there was no differential intersensory facilitation related to laterality.

An irrelevant sound can facilitate response to a visual stimulus (Bernstein, Pederson, \& Schurman, 1972; Morrell, 1968; Simon \& Craft, 1970). This effect does not depend on S's response strategy or on well known modality differences in simple RT (Taylor, 1973). Rather, the behavioral evidence combines to suggest that the facilitation directly reflects neural constraints on sensory interaction. It has been argued, in particular, that there is a need to assume that sound can modulate ongoing messages in the retino-cortical projection system (Taylor, 1973). This hypothesis has recently received support from single-unit recordings in the visual cortex and superior colliculus in response to round window polarization (Schwartzkroin, 1973).

Studdert-Kennedy \& Shankweiler (1970) used a paradigm requiring $S$ to attend to and recall simultaneous dichotic speech. They found that if the aural components of the input conveyed language information, stimuli presented at the right ear were processed more efficiently than those presented at the left ear. In the same paper, they argue that auditory systems common to both hemispheres are able to extract simple auditory parameters. Thus, acoustic laterality arises because of an interaction of the message and where it is analyzed-rather than to simple superiority of the left cerebral auditory hemisphere. Taylor (1973), using a reaction time (RT) task and several classes of aural stimuli (such as phoneme segments and music), all of which were incidental to S's task, found no left-right or other differential aural laterality effect on visual RT performance. These data tend to suggest that Ss were not processing the sound at a high level, in spite of the fact that it conveyed meaningful information. However, the visual stimuli were presented foveally, and hence were represented in both cerebral hemispheres directly. It was, therefore, not possible to determine if any underlying laterality of effect existed for different ear/visual field combinations.

\footnotetext{
*This work was supported by Grants A-7811 and E-3329 from the National Research Council of Canada to the second author. The senior author conducted this work as partial fulfillment of the requirements for an undergraduate course at
} Memorial University.
In this experiment, we examine the effect on RT of combinations of stimuli presented in left or right visual fields and aural stimuli presented monaurally left or right, or binaurally.

\section{METHOD \\ Subjects}

The Ss were 25 graduate and undergraduate students enrolled at Memorial University.

\section{Stimuli}

Visual stimuli were digits presented on a cathode ray tube (CRT). Each stimulus subtended a horizontal visual angle of $18 \mathrm{~min}$ and a vertical angle of $30 \mathrm{~min}$, plus or minus $10 \mathrm{~min}$, as S moved his head. Aural stimuli were $90-\mathrm{dB}$ SPL noise bursts filtered to overlap the speech spectrum, and delivered to $S$ through stereo headphones. A Hewlett-Packard 2114 computer controlled all events within a session, including timing and presentation of stimuli. This system is described in more detail by Taylor (1972).

\section{Procedure}

Each trial consisted of three consecutive displays. First, a reference digit and a target digit were displayed on the CRT, the former always being located to the left of the latter. Second, the target digit was removed and the reference digit alone remained. The third display consisted of the test digit being displayed approximately $2 \mathrm{deg}$ to the right of the reference digit (half of the trials) or $2 \mathrm{deg}$ to the left of the reference digit.

The aural stimuli were completely incidental to the task of S. The task was to determine if the target and test digits were the same. If so, S was to respond "same" by pressing a button under his middle finger. If they were not the same, $\mathrm{S}$ was to respond "no" by pressing a button under his index finger.

\section{Temporal Scaling}

Boredom or unnecessary double checking, as well as inattention, can produce excessively long or erratic RT distributions. One attempt to reduce these sources of inhomogeneity is to discard responses which take too long. That procedure fails to attack the source of the inhomogeneity because it fails to inform $S$ on those trials in which boredom or inattention occur that such behavior constitutes an error. Similarly, an arbitrary statistical criterion for what is "too long" is fallible in rough proportion to the frequency of such errors, which have not been estimated directly. The procedure used in this experiment was to establish a time limit for responding and to adjust exposure durations of the stimulus arrays on each trial, for each S, at a value near the upper end of his cumulative reaction-time distribution. Thus, the time scale of trial and permissible RTs were altered throughout a session. Further, immediate feedback was given: When an $\mathrm{S}$ erred, either by not responding within the time limit or by making an incorrect response, a large $X$ appeared in the center of the CRT screen. When the $S$ was correct, his RT for that trial was displayed immediately in the lower left corner of the screen. The $S$ initiated each trial by pressing both buttons, thereby insuring that the intertrial interval met his changing criterion of readiness. The unit for time scaling was a standard interval (SI), defined on Trial $n$ as the lesser of $1,500 \mathrm{msec}$ or the S's cumulative mean $\mathrm{RT}+3 \mathrm{SD}$. The three visual displays presented on Trial $\mathrm{n}+1$ were each exposed for $\mathrm{SI}_{n}$, and the time limit for responding during the test interval of $n+1$ was also $S I_{n}$. The SI was recalculated on each trial by the computer.

\section{Design}

The onset of sound was set at $5 \%$ of the elapsed interval 
throughout a session. Noise terminated when the trial terminated. either when the time limit has elapsed or when $S$ responded. There were four "sound" conditions. monaural left. monaural right. binaural. and no noise. and two visual stimulus locations. left of fixation and right of fixation. All events were randomized within a session. with the constraint that no one condition was allowed to occur more than $10 r^{\circ}$ more or less frequently than any other condition.

A session was programmed to terminate after the dual criteria of $20 \mathrm{~min}$ and 250 trials had been satisfied. Each $\mathrm{S}$ was tested in two sessions with a brief rest period.

\section{RESULTS}

The variables of sound condition (noise-left, noise-right. noise-binaural, quiet) by visual location (left, right) by blocks (Sessions 1. 2) were combined in an overall analysis of variance of the mean RTs. The highest order (three-way) interaction was used as the error term. The only significant results were the simple main effects of sound condition. visual location (digits on the right were faster by $14 \mathrm{msec}$ than those on the left, $F=25.3$. $\mathrm{df}=1.24 . \mathrm{p}<.001$ ), and practice (Session 2 was faster overall by 32 msec than Session 1 ).

Since the quiet trials provide a control baseline for estimating the amount of intersensory facilitation, a second analysis of variance was run on the difference scores. Each datum for this analysis was obtained by subtracting the mean noisy-trial RT from the mean quiet-trial RT. This reduces the number of levels of the sound condition variable from four to three, but otherwise the analysis was exactly analogous to the one described above. There was a practice effect, with $38.8 \mathrm{msec}$ mean facilitation of RT in Session 1. and $29.3 \mathrm{msec}$ facilitation in Session $2(\mathrm{~F}=7.4, \mathrm{df}=1,24$, $p<.025)$. The principal finding was that binaural sound facilitated visual RT more (by $46 \mathrm{msec}$ ) than either left or right ear monaural sound presentation $(\mathrm{F}=11.8, \mathrm{df}=$ 2.48. $\mathrm{p}<.001$ ), the two monaural conditions not producing any difference in facilitation (28.3 and
27.9 msec. respectively). To other results were significant in this analysis.

\section{DISCUSSION}

The present results show that the facilitation of visual RT by an irrelevant sound does not vary with the location of the visual stimulus or with the ear into which the sound is introduced. This failure to find any evidence of a laterality effect-while at the same time picking up a clear intersensory facilitation of reaction time-supports an earlier conclusion that the phenomenon has its origins very early in the sensory nervous system, prior to complex processing of either the visual or auditory stimulus by the brain (Taylor, 1973).

It is also clear that two ears are better, in this respect, than one. Summation may also imply a primitive sensory locus within the nervous system: in particular. it now seems plausible that input from the two ears functionally converges on the visual projection system. Since it has been shown that the result of aural or vestibular stimulation is to inhibit single unit activity in the superior colliculus and to facilitate activity of cells in the visual cortex, the possibility arises that the convergence may actuate either excitatory or inhibitory effects on neural contrast analy'sis (Schwartzkroin. 1973).

\section{REFERENCES}

Bernstein, I H. Pederson, N. N., \& Schurman, D. L. Intersensory versus intrasensory contingent information processing. Journal of Experimental Psychology, 1972.94, $156-161$.

Morrell. L. K. Temporal characteristics of sensory interaction in choice reaction time. Journal of Experimental Psychology, $1968,77,14-18$

Schwartzkroin, P. A. Effects of round window stimulation on unit discharges in the visual cortex and superior colliculus. Experimental Brain Research, 1973, 17, 527-538.

Simon, J. R., \& Craft. J. L. Effects of an irrelevant auditory stimulus on visual choice reaction time. Journal of Experimental Psychology, 1970.86, 272-274.

Studdert-Kennedy, M., \& Shankweiler, D. Hemispheric specialization for speech perception. Journal of the Acoustical Society of America, 1970, 48, 579-594.

Tavlor. R. L. Coglab: A computer system designed for human research. Behavior Research Methods \& Instrumentation. $1972,4,94-95$.

Taylor, R. L. An analysis of sensory interaction. Neuropsychologia, 1973, in press.

(Received for publication October 18, 1973.) 